\title{
remblais autoroutiers sur ballastières remblayées
}

\author{
par \\ J.P. Cudennec \\ Laboratoire Régional des Ponts et Chaussées, Le Bourget \\ J.P. Gigan \\ Laboratoire Régional des Ponts et Chaussées, Le Bourget \\ G. Chartier \\ L. C.P.C., Paris
}

RESUME : Plusieurs tronçons de l'Autoroute A 86 autour de Paris, comportant des remblais et
des ouvracres de soutènement, traversent d'anciennes ballastières remblayées. Les matériaux de
comblement, d'une dizaine de mètres d'épaisseur, constituent un niveau compressible dont le
comporterent est difficile à appréhender par les méthodes classiques. Dans les exemples ex-
posés, le recours à la mise en oeuvre de plates-formes préalables ou de préchargement ins-
trumentés a palliéaux insuffisances des études prévisionnelles. Les résultats ont montré que
dans ce type de matériau on obtient des tassements très variables selon les sites, mais se
stabilisant toujours en ouelques mois. On note une nette amélioration du comporterent mécanioue.

PROBLEMES LIES A LA CONSTRUCTIBILITE DE BALLASTIERES REMBLAYEES EN REGION PARISIENNE

Parmi les rares zones non urbanisées autour de Paris, les anciennes exploitations de sables et graviers dans les plaines alluviales de la Seine et de la Marne constituent des points de passage privilégiés mis à profit par les tracés autoroutiers.

Le comblement de ces exploitations a été généralement effectué sans précaution sur le choix et le mode de mise en oeuvre des matériaux de remblai, on y trouve une part importante de gravois et de déblais de terrassement mais également un pourcentage non négligeable de déchets industriels et ménagers.

Les études prévisionnelles de construction d'ouvrage, de remblais de divers types et de soutènement sur ces matériaux se heurtent actuellement à l'absence d'une méthodologie spécifique. En effet les méthodes d'étude des sols compressibles ne peuvent s'appliquer du fait de l'impossibilité de prélever des échantillons représentatifs pour analyse en Laboratoire.

Sur l'Autoroute A 86 (fig. 1), pour laquelle ces problèmes se sont présentés à plusieurs reprises, les moyens de reconnaissance géotechniques mis en oeuvre sont essentiellement des sondages destructifs à la tarière en gros diamètre et des forages pressiométriques.

Des tentatives d'utilisation du pénétromètre statique se sont avérées inefficaces du fait du grand nombre de refus sur blocs.

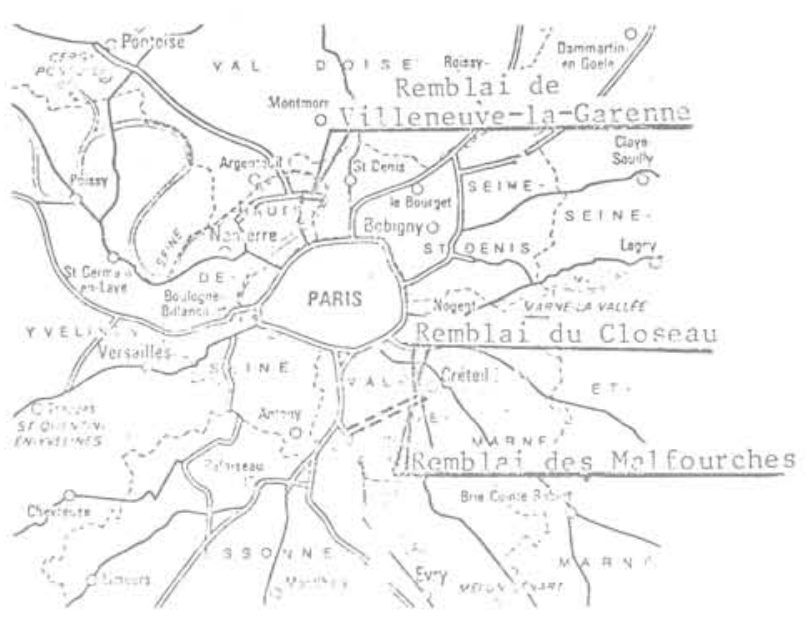

Fig. 1 - plan de situation des remblais

Les informations obtenues permettent de prévoir les conditions de portance initiales du sol; mais elles sont insuffisantes pour estimer convenablement I'amplitude des tassements, leur évolution dans le temps et l'amélioration du comportement sous l'effet d'un chargement.

La connaissance de ces renseignements est pourtant, dans de nombreux cas, indispensable 
dès le stade de l'élaboration des projets pour juger de la faisabilité de remblai de grande hauteur, de l'établissement des plannings de construction, de l'opportunité de la prise en compte d'efforts parasites sur les fondations profondes.

Dans les trois exemples cités ci-après concernant la construction de remblais de différentes hauteurs entre ouvrages d'art, l'influence des paramètres mal définis par la reconnaissance géotechnique traditionnelle était déterminante pour le projet. Les précisions nécessaires ont été obtenues par la mise en oeuvre de plates-formes d'essai préalables.

Dans deux cas, ce sont des sections du remblai définitif édifiées au moment des études qui ont étê instrumentées; dans l'autre il. s'agit d'un remblai de préchargement mis en place avant l'édification d'une culée en terre armée.

\section{LES REMBLAIS DE VILLENEUVE-LA-GARENNE}

\section{- Le site, le projet, les scls}

Ce tronçon de 1'Autoroute A 86 se situe au Nord de Paris dans le méanđre de la Seine dit "Boucle de Gennevilliers" entre la voie rive gauche de Seine et la Nationale 186.

L'Autoroute est en remblai de 4 à 7 mètres de hauteur, limité pour des raisons d'emprise, par des ouvrages de soutènement. La voirie locale est rétablie par plusieurs passages inférieurs.

La géologie du site se caractérise par une couverture alluvionnaire d'une dizaine de mètres d'épaisseur au-dessus du substratum constitué par le calcaire de Saint-Ouen ou les Sables de Beauchamp. Le niveau de la nappe phréatique, déprimé par les pompages industriels se situe vers $7 \mathrm{~m}$ de profondeur. Les alluvions ont été exploitées, les dernières extractions remontant à une dizaine d'années.

\section{- L'étude préalable, ses conclusions}

La reconnaissance de la nature et du comportement mécanique des remblais a été menée à partir de forages à la tarière Highway et de forages pressiométriques selon des profils distants d'une cinquantaine de mètres.

Les_matériaux_rençontrés sont d'une très grande diversitê : limons plus ou moins organiques, déchets industriels (cendres, éléments métalliques, hydrocarbures...) résidus de démolitions (gravois, bois). De gros volumes du type carcasse d'automobile ou

appareil ménager n'ont pas été rencontrés et leur probabilité d'existence a été considérée comme faible.

Les_résultats_pressicimétriquues présentent natureliement une forte dispersion. Les moyennes géométriques sont les suivantes :
- pression limite

$\mathrm{Pl}=340 \mathrm{KPa}$ écart type relatif $1,7 \%$ - Module pressiométrique

$\mathrm{E}=2500 \mathrm{KPa}$ écart type relatif 1,7

"Ceci signifie que $68 \%$ des valeurs sont comprises entre $\frac{340}{1,7}$ et $340 \times 1,7 \mathrm{KPa}$.

La_capacité_portante initiale du sol n'autorise pas l'édification d'ouvrages de soutènement rigides (murs en béton armé) fondés superficiellement.

L'édification directe de murs en terre armée, qui ne pouvait être envisagée que moyennant une construction par étapes, a été également écartée à défaut de moyens de prévisions fiables des tassements (amplitude et vitesse) et de I'amélioration du comportement mécanique.

Des fondations profondes sont indispensables pour les ouvrages. Leur dimensionnement nécessite de connaitre l'évolution dans le temps des déformations des remblais, susceptibles de provoquer des efforts parasites.

Pour lever ces incertitudes, la réalisation d'un rerblai rréalable a 'té retenue.

\section{- L'intrumentation du remblai, ses résultats} Le_remblai a été équipé de 3 profils transversaux de 3 tassomètres de surface et de 2 tubes inclinométriques ancrés dans le substratum. (fig, 2). Il a été monté en première phase jusqu'à une hauteur de 4 à $4,5 \mathrm{~m}$ en un mois, puis élargi latéralement en deuxième phase (fig. 3 ). Les inclinomètres se situant respectivement en pied du talus lère phase et du talus 2ème phase.

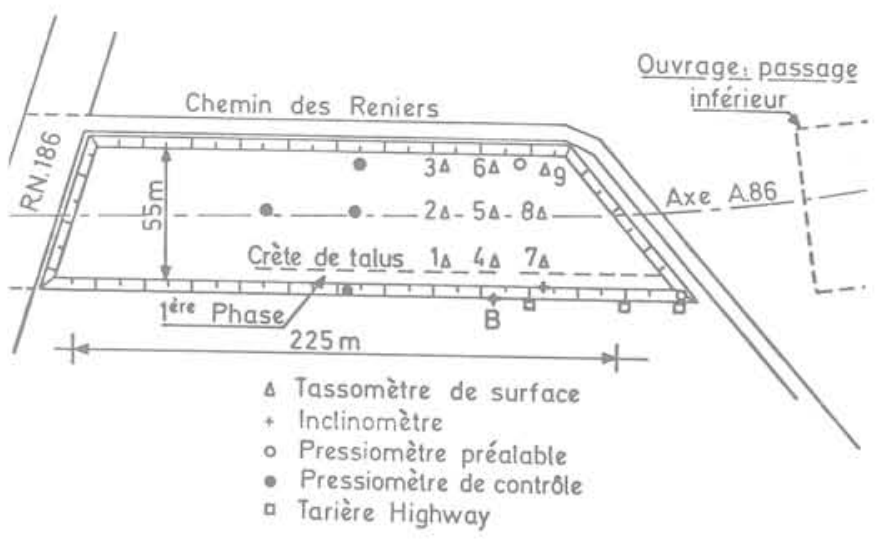

Fig. 2 - Implantation et instrumentation du remblai de Villeneuve.

L'amplitude des_tassements (fig 4) varie entre 22 et $2 \overline{9}$ centimètres. Ils se manifestent rapidement : $40 \%$ du tassement est acquis en cours de chargement et $90 \%$ en moins de 2 mois, 


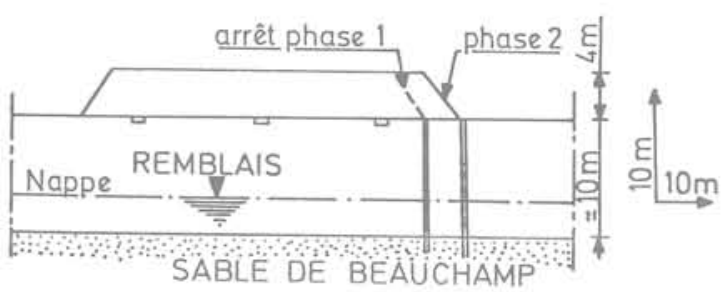

Tube Inclinomètrique

$\square$ Tassomètre

Fig. 3 - Coupe transversale du remblai de Villeneuve-La-Garenne

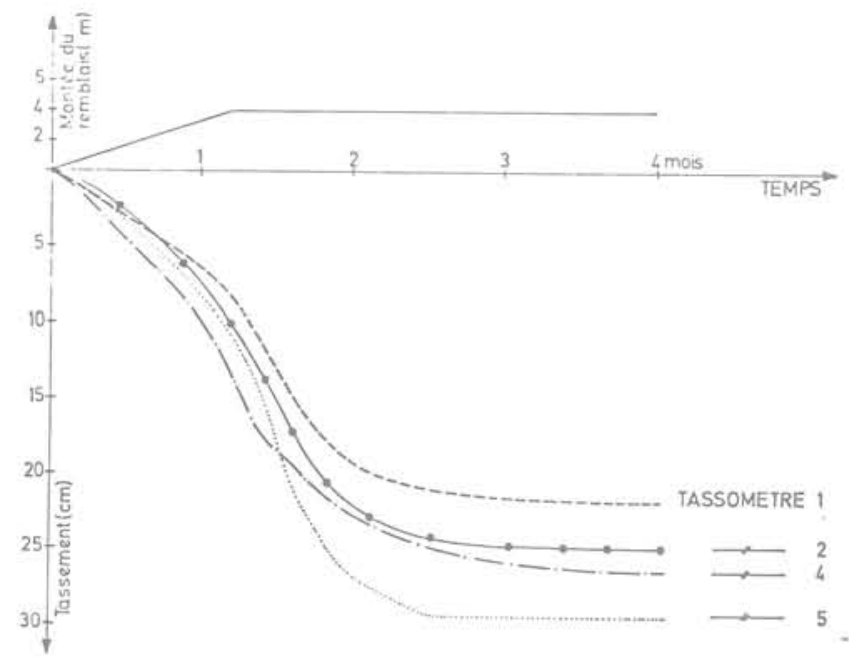

Fig. 4 - Evolution des tasserents du remblai de Villeneuve-la-Garenne

L'évolu est représentée sur la figure 5 en fonction de la profondeur et à différentes dates.

On constate que leur amplitude reste inférieure à $2 \mathrm{~cm}$ soit environ 5 \% de la valeur des tassements. La stabilisation est également observée deux mois après l'exécution de la deuxième phase de chargement.

- L'améliororation_du﹎ongomportement_mécanigue a été mesurée par 4 sondages pressiométriques de contrôle. Les moyennes géométriques obtenues sont les suivantes :

-pression limite,

$\mathrm{P} 1=720 \mathrm{KPa}$ écart type relatif 1,7

-module pressiométrique

$\mathrm{E}=4600 \mathrm{KPa}$ écart type relatif 2

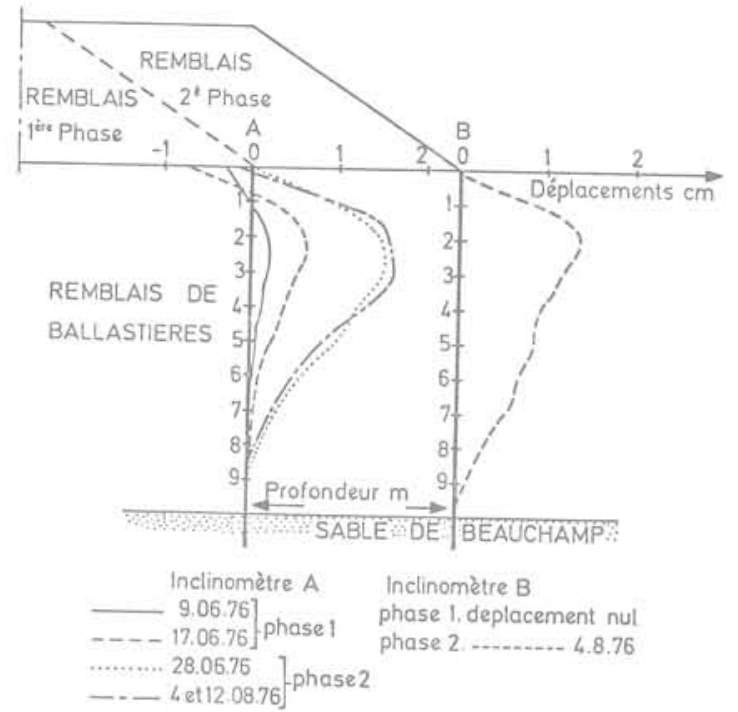

Fig. b - Déplacements horizontaux du remblai de Villeneuve-la-Garenne

Les pressions limites et les modules ont donc été multipliés par un facteur de 2 en moyenne, mais la dispersion reste forte.

Un nombre plus élevé de forages réalisés par la suite sur ce tronçon autoroutier (110 essais) a confirmé ce résultat.

- Les enseignements tirés de 1'expérimentation

Les tassements sous un remblai peuvent être estimês selon les règles pressiométriques par l'expression :

$W(m)=\alpha \times \frac{\mathrm{P}}{\mathrm{E}} \times \mathrm{h}$

$\mathrm{p}$ est la contrainte due au remblai en Kpa

$\mathrm{E} \quad$ le module pressiométrique en $\mathrm{KPa}$

$\alpha$ un coefficient dénendant de la nature du sol et de la céorétrie du remblai.

h épaisseur du sol compressible en mètre. Les tassements mesurés sont retrouvés par le calcul à condition d'adopter sur ce site une valeur de $\alpha^{-}=1$.

L'amélioration des_caractér. gistrée a permis de fonder superficiellement les ouvrages de soutènement : moyennant un décaissement partiel des remblais, les murs en terre armée ont été exécutés sans aléas jusqu'à une hauteur de 7 mètres.

Les fondations profondes ont pu être effectuées après stabilisation totale, dans des délais compatibles avec un déroulement normal du chantier, tout en évitant le surdimensionnement qu'aurait entrâ̂né la prise en compte des poussées latérales.

La consolidation préalable a en outre 
facilité les conditions de foration des pieux et barrettes.

\section{LE REMBLAI DU "CLOSEAU" A CRETEIL}

\section{- Le site - Le projet - Les sols}

Ce remblai, dont la hauteur maximale atteint 10 mètres, supporte une voie de raccordement provisoire de 1'Autoroute A 86 sur la RN 6 à Créteil.

Dans cette zone, les Alluvions anciennes de la Seine ont également fait 1'objet d'exploitations récentes. Le terrain d'assise est donc constitué par 8 à $9,00 \mathrm{~m}$ de remblais de comblement reposant sur le substratum formé par le marno-calcaire de Saint-Ouen. La nappe phréatique est rencontrée à $3 \mathrm{~m}$ de profondeur.

- L'étude préalable - Ses conclusions

Une campagne d'une vingtaine de sondages à la tarière en 0,60 de diamètre a permis de reconnaltre la nature des remblais de comblement. On rencontre des matériaux de terrassement, des débris de démolitions (avec un pourcentage de blocs important) et des déchets industriels et ménagers atteignant 10 \% du volume total.

Il faut noter que ce moyen d'investigation devient relativement imprécis sous le niveau de la nappe phréatique où le pourcentage de récupération est faible.

Le comportement mécanique testé au pressiomètreafourni les valeurs moyennes suivantes:

- pression limite

$\mathrm{Pl}=340 \mathrm{KPa}$

- Module pressiométrique

$\mathrm{E}=2200 \mathrm{KPa}$

Les caractéristiques mesurées, complétées par l'expérience du remblai de Villeneuve dont nous disposions, conduisaient à des tassements prévisibles supérieurs à $50 \mathrm{~cm}$. Ces conditions étaient particulièrement contraignantes au droit de la culée implantée sur la limite de l'exploitation côté RN6. Ceci a conduit à prévoir un mur de front en terre armée indépendant de la pile-culée reprenant les réactions d'appui du tablier.

L'édification directe d'un tel massif nécessitait une construction par étapes. Cette solution n'a pas été retenue compte tenu de l'incertitude sur les capacités d'amélioration du sol d'assise et les tassements différentiels, (on pouvait craindre en particulier des dificultés d'assemblage et d'alig̣nement des écailles en béton armé entre phases).

Parmi diverses solutions d'amélioration du sol, le préchargement a été retenu.

- L'instrumentation du remblai, ses résultats Le problème essentiel étant le suivi des tassements (amplitude et évolution en fonction du temps) seuls des tassomètres de surface ont été mis en place, 11 cellules ont été réparties sous l'emprise du remblai (fig. 6).

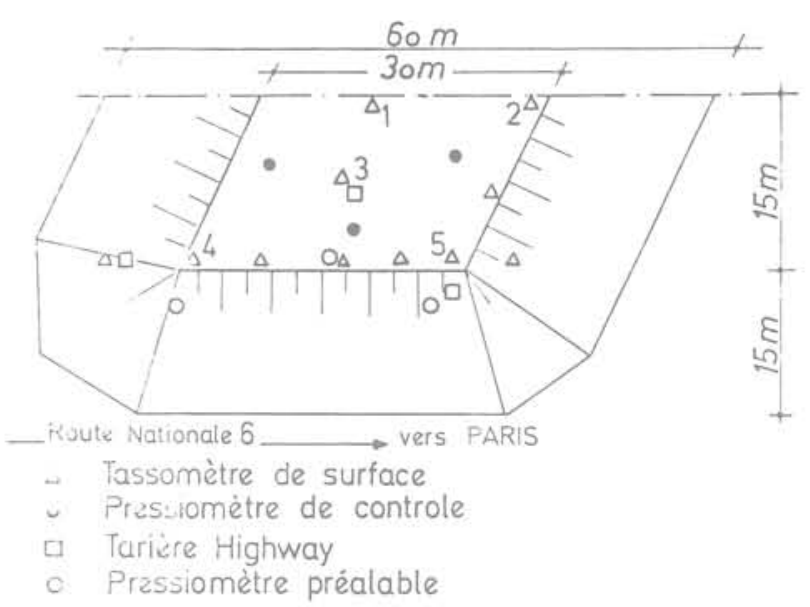

Fig. 6 - Implantation et instrumentation du remblai du closeau

La montée du remblai (fig. 7) s'est déroulée en trois phases jusqu'à une hauteur de $11 \mathrm{~m}$ par rapport au terrain naturel (hauteur supérieure de $3 \mathrm{~m}$ à celle du massif en terre armée). Cependant, lors de la mesure initiale du tassement, une hauteur de $1,50 \mathrm{~m}$ de remblai était déjà mise en oeuvre.

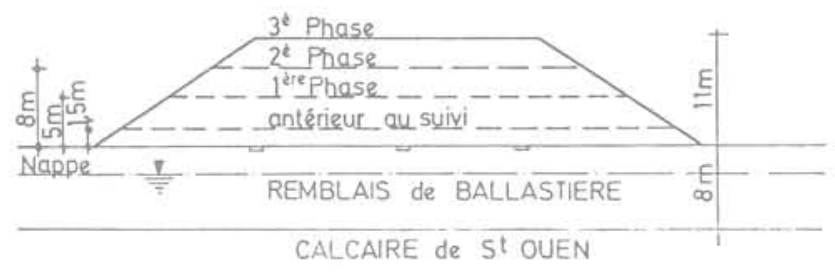

Fid. 7 - Coupe transversale du remblai du Closeau

Les_résultats_des_mesures (fig. 8) montrent que le tassement final est compris entre 15 et $20 \mathrm{~cm}$. Pour la première phase de remblai, d'une hauteur de 3,5 mètres, le tassement a été d'environ $5 \mathrm{~cm}$, alors que pour la deuxième phase limitée à 3 mètres, il a été nettement plus élevé et a atteint 12 centimètres. Sous la troisième phase, les tassements sont par contre inférieurs, ce qui s'explique par l'amélioration des caractéristiques du terrain et la diffusion des contraintes.

Les tassements s'effectuent en grande partie simultanément au chargement, une stabilisation presque totale est obtenue après 2 à 3 semaines. 


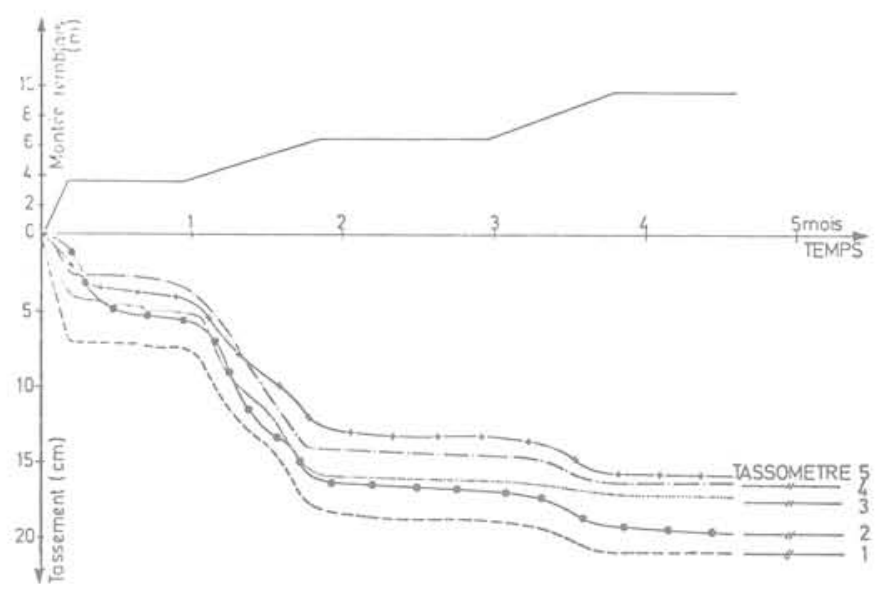

Fid. 8 - Evolution des tassements du remblai du Closeau

L' amélior a été contrôlée par trois forages pressiométriques effectués après déchargement. Les valeurs moyennes mesurées sont doublées par rapport aux valeurs initiales :

- pression limite : PI $=740 \mathrm{KPa}$

- Module pressiométrique : $E=4500 \mathrm{KPa}$

- Les enseignements tirés de l'expérimenta tion

Si on applique le même raisonnement que pour le remblai de Villeneuve-La-Garenne, on constate que pour faire correspondre les tassements mesurés aux calculs par la méthode pressiométrique, le coefficient $\alpha$ doit être voisin de 0,3 .

L'amélioration des caractéristiques a permis l'édification du massif en terre armée à sa cote définitive en une seule phase avec un coefficient de sécurité de 2 , suffisant pour ce type d'ouvrage. Toutefois, des tassements de l'ordre de $10 \mathrm{~cm}$ ont été mesurés lors de la construction.

\section{LE REMBLAI DES "MALFOURCHES" A CRETEIL}

- Le projet, le site

Ce remblai s'intègre dans l'échangeur du Carrefour Pompadour sur 1'autoroute A 86 à créteil.

Il assure la jonction entre les ouvrages de franchissement de la RN 6 et des voies SNCF PARIS-LYON. Sa hauteur atteini $13 \mathrm{~m}$.

Sur le site de cet échangeur qui présente des conditions géotechniques similaires au site du "Closeau" situé $500 \mathrm{~m}$ au Nord plusieurs autres remblais doivent être édifiés.

- L'étude préalable, ses répercussions sur le projet

Les remblais de comblement, mis en place en 1976 sont essentiellement des limons de découverte et des marnes, le pourcentage de matériaux de démolitiors ne dépassant pas $20 \%$.

Les caractéristiques pressiométriques sont particulièrement faibles :

- pression limite moyenne : 220KPa

- module pressiométrique moyen : $2000 \mathrm{KPa}$

De telles caractéristiques paraissaient a priori peu favorable à l'édification d'ouvrages en terre de grande hauteur. Une augmentation des valeurs des pressions limites de 250 \% pour un remblai et de $400 \%$ pour un massif en terre armée était en effet nécessaire dans le cas présent en phase finale de construction.

Les résultats obtenus sur le remblai du "Closeau" ainsi que l'existence à proximité du site d'une butte paysagère de $20 \mathrm{~m}$ de hauteur, tendaiert cependant à montrer que moyennant une montée progressive pilotée par un contrôle rigoureux, on pouvait atteindre la cote prévue avec une sécurité convenable. La réalisation d'un remblai préalable instrumenté a donc été đécidée dans cette zone des l'alfourches. Les résultats devant constituer une référence nrécieuse pour l'enserble des autres remblais de l'Echanceur.

- L'instrumentation du remblai, ses premiers résultats

L'équipement mis en place (fig. 9 et 10) comporte :

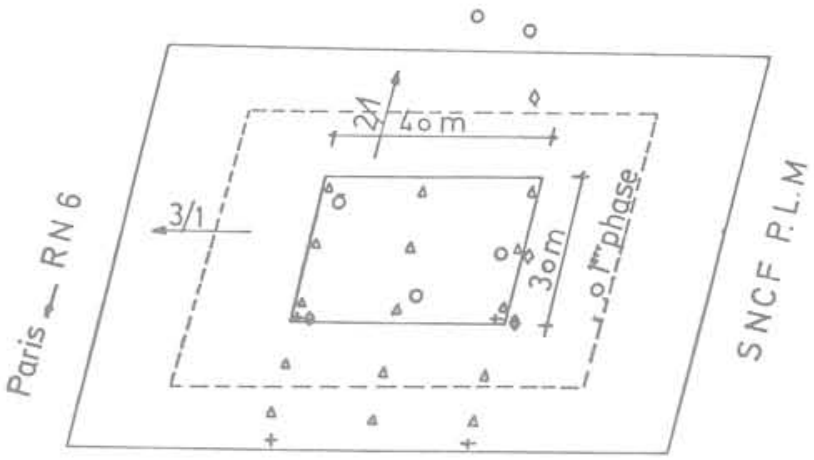

$\triangle$ Tassomètre de surface

- Pressiomètre

+ Inclinomètre

- Tassomètre de profondeur

Ficc. 9 - Implantation et instrumentation du remblai des Malfourches

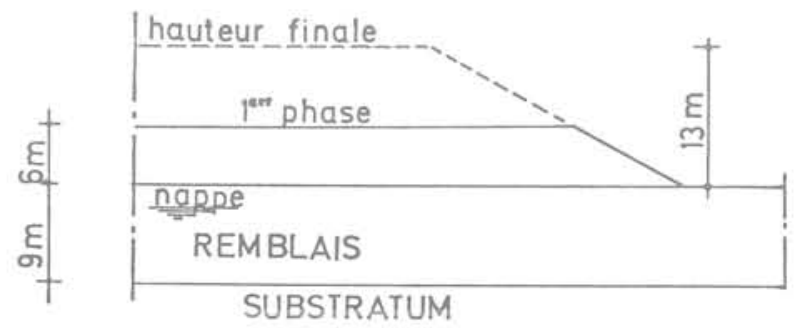

Fiq. 10 - Coupe transversale du remblai des Malfourches 
- 3 profils de 6 tassomètres de surface

- 4 tassomètres de profondeur (repères multiples de tassement)

- 4 tubes inclinométriques

- 4 sondes de mesure de la pression interstielèlle.

En Novembre 1980, une première étape de chargement d'une hauteur de 6 mètres avait été effectuée. Le tassement moyen a atteint 14 centimètres, mais sa stabilisation, plus lente que pour le remblai du "Closeau", n'était pas entièrement acquise au bout de six semaines.

Des résultats plus complets du suivi de cet ouvrage devraient être disponibles lors des journées en Mars 1981.

\section{CONCLUSION}

L'hètérogénéité des remblais de comblement de ballastières impose une forte densité de points de reconnaissance.

Des sondages carottés ou destructifs de gros diamètre sont nécessaires pour déterminer leur nature et apprécier le pourcentage de matières organiques susceptibles d'évolution dans le temps. Une approche du comportement mécanique ne peut être fournie que par des essais in situ (forages pressiométriques ou pénétrométriques en 1'absence d'un pourcentage élevé de blocs).

Sur les trois sites ayant fait l'objet d'un suivi lors du chargement par un remblai, le pourcentage de matières organiques était faible. Les matériaux de comblement, essentiellement constitués de déblais de fouilles et de débriśs de démolition présentaient des caractéristiques mécaniques très faibles et comparables d'un site à l'autre.

Les résultats obtenus ont montré que l'amplitude des tassements est le paramètre le plus difficile à prévoir : on constate que sur le site du closeau, pour une hauteur de chargement d'une dizaine de mètres les tassements sont plus faibles qu'à Villeneuvela-Garenne où le chargement n'a atteint que 4,5 mètres.
Les vitesses de tassement ont sté capides et se sont stabilisées en quelques mois. Ce phénomène est certairement dû à $I^{\prime}$ absence de niveaux argileux de forte épaisseur et aux propriétés drainantes des matériaux de démolition.

L'amélioration du comportement mécanique a été très nette.

Aucun désordre n'a été constaté sur les ouvrages depuis leur construction, ce qui confirme l'efficacité de la technique utilisée. Toutefois, les différences de comportement d'un site à l'autre enregistréeslors du suivi, notamment en ce qui concerne les tassements, ainsi que le faible nombre d'exemples disponibles ne permettent pas de généraliser les résultats obtenus.

\section{BIRLIOGRAPHIE}

CAP.TIER G. (1981), Comportement céotechnique des résidus urbains. IIème journées nationales qéotechniques, Nantes.

CARTIEP C., LONE N.T., POUGET P., BARCILLAT R., CUDENNEC J.P., Déchets urbains et pneumaticues usagés en oénie civil. dixième congrès international de mécanique des sols. Stockholm, Juin 1981

SOWERS, G.F. (1968). Foundation problems in sanitary Landfills, ASCE, J of the SED, (94) $\mathrm{n}^{\circ}$ SAI, 103-116. 